\title{
Enfermedad cardiovascular y metabólica en pacientes infectados por el virus de la inmunodeficiencia humana
}

\author{
William Alejandro Camargo-Ariza* \\ Silvia Juliana Galvis-Blanco* \\ Édgar Felipe Gualdrón-Rincón** \\ Jorge Mario Palmezano-Díaz ${ }^{* * *}$
}

\begin{abstract}
*Estudiante de IX nivel de Medicina. Universidad Industrial de Santander. Escuela de Medicina. Facultad de Salud. Bucaramanga. Santander. Colombia. **Estudiante de VII nivel de Medicina. Universidad Industrial de Santander. Escuela de Medicina. Facultad de Salud. Bucaramanga. Santander. Colombia.

***Residente de Medicina Interna. Universidad Industrial de Santander. Servicio de Medicina Interna. Hospital Universitario de Santander. Bucaramanga. Santander. Colombia.

Correspondencia: William Alejandro Camargo Ariza. Dirección: Carrera 23 \# 31-63 Apto 504. Bucaramanga. Santander. Colombia. Teléfono: (+57) 3125847934. Correo electrónico: willyamcgo@hotmail.com
\end{abstract}

\section{RESUMEN}

Desde la instauración de la terapia antiretroviral, la morbimortalidad asociada al síndrome de la inmunodeficiencia adquirida ha disminuido drásticamente y la infección por el Virus de la Inmunodeficiencia Humana se ha convertido en una enfermedad crónica. Sin embargo, con la introducción del tratamiento antiretroviral para el control de la enfermedad se ha observado un aumento en la prevalencia de enfermedad cardiovascular en este grupo, convirtiéndose éste en un motivo de especial preocupación. Consecuentemente, la evaluación y prevención de la enfermedad cardiovascular se ha tornado de suma importancia y debe evaluarse de manera individual y rutinaria en estos pacientes. El objetivo de este artículo es hacer una revisión de las principales complicaciones cardiovasculares y metabólicas con el fin de consolidar los posibles hallazgos a los que se enfrenta el clínico al momento de un abordaje integral de un paciente seropositivo para el virus de inmunodeficiencia humana. MÉD.UIS. 2017;30(2):45-54.

Palabras clave: Terapia Antirretroviral Altamente Activa. Enfermedades Cardiovasculares. Enfermedades metabólicas. VIH.

\section{Cardiovascular and metabolic disease in patients with human immunodeficiency virus infection}

\section{ABSTRACT}

Since the introduction of antiretroviral therapy, morbidity and mortality associated with Acquired Immunodeficiency Syndrome has drastically decreased and human immunodeficiency virus infection has become a chronic disease. However, with the introduction of antiretroviral treatment for the control of the disease, an increase in the prevalence of cardiovascular disease in this group has been observed, making this a reason of particular concern. Consequently, the evaluation and prevention of cardiovascular disease has become of paramount importance and should be evaluated individually and routinely in these patients. The objective of this article is to make a review of the main cardiovascular and metabolic complications in order to objectify the possible findings that the clinician faces at the moment of an integral approach to the seropositive patient for human immunodeficiency virus. MÉD.UIS. 2017;30(2):45-54.

Keywords: Antiretroviral Therapy Highly Active. Cardiovascular Diseases. Metabolic Diseases. HIV.

¿Cómo citar este artículo?: Camargo-Ariza WA, Galvis-Blanco SJ, Gualdrón-Rincón EF, PalmezanoDíaz JM. Enfermedad cardiovascular y metabólica en pacientes infectados por el virus de la inmunodeficiencia humana. MÉD.UIS. 2017;30(2):45-54. 


\section{INTRODUCCIÓN}

Desde 1987, cuando la Agencia de Alimentos y Medicamentos de los Estados Unidos (FDA, por sus siglas en inglés) aprobó la Terapia Antiretroviral (TAR) esta ha llevado a una drástica reducción del $15 \%$ en la morbimortalidad asociada al Síndrome de Inmunodeficiencia Adquirida (SIDA), determinando que la infección por el Virus de la Inmunodeficiencia Humana (VIH) sea una enfermedad crónica. Así mismo, se ha observado que con el tratamiento para el control de la enfermedad, ha aumentado la prevalencia de varias enfermedades crónicas, entre ellas la Enfermedad Cardiovascular (ECV) la cual fue descrita por primera vez por Sir Keith Henry y Colaboradores en 19981, desde entonces se ha convertido en motivo de especial preocupación debido a los cambios metabólicos inducidos por la TAR, la alta prevalencia de factores de riesgo cardiovasculares (entre 35,2 y $15,8 \%$ en Colombia) ${ }^{10}$ y procesos inflamatorios que promueven aterosclerosis acelerada en individuos infectados por el $\mathrm{VIH}^{2}$. A su vez se ha propuesto que la carga viral, los factores inmunológicos y la inflamación relacionadas a la discontinuación del tratamiento, contribuyen al aumento del riesgo cardiovascular ${ }^{3}$, todo esto ha llegado a indicar que los factores de riesgo cardiovasculares modificables son más prevalentes en los pacientes infectados por el VIH.

Desde 1990, cuando se introdujo y generalizó en el mercado la Terapia Antirretroviral de Gran Actividad (TARGA), ha generado un descenso en la mortalidad en estos pacientes. Sin embargo, existe una creciente preocupación sobre las complicaciones metabólicas y cardiovasculares asociadas con el VIH y la TARGA. Este efecto se debe a la similitud de la región catalítica de la VIH proteasa (diana de los inhibidores de las proteasas) con dos proteínas humanas encargadas de regular el metabolismo de los lípidos: la proteína 1 ligadora del ácido retinoico citoplasmático y la proteína relacionada con el receptor de la lipoproteína de baja densidad ${ }^{4,13}$, generando una alteración en el metabolismo de los lípidos.

El Programa Conjunto de las Naciones Unidas sobre el VIH/SIDA (ONUSIDA) estimó en junio de 2015 que había 36,9 millones de personas infectadas con el $\mathrm{VIH}$ en el mundo. El número de personas con el virus sigue aumentando, en gran parte debido a un mayor acceso a la TAR, aumentando la expectativa y calidad de vida. Este aumento ha sido del 84\% desde el 2010 en países en vía de desarrollo, resultando en un importante descenso en la mortalidad por VIH/SIDA, y disminuyendo en un $35 \%$ la incidencia de la infección por el virus desde inicios del siglo pasado ${ }^{5-7}$.

En América Latina la infección por VIH se presenta principalmente en poblaciones vulnerables, en especial hombres que tiene sexo con hombres y trabajadores sexuales. El número de primoinfecciones por VIH en 2014 bajo en un 17\% a compraración del año 2000 , así mismo hubo una disminución del 31\% en la mortalidad relacionada con el SIDA desde el mismo año ${ }^{8}$.

En Colombia, se inició el proceso de notificación de casos y muerte por VIH/SIDA desde 1983. Actualmente el Ministerio de Salud y Protección Social a través del Sistema de Vigilancia Epidemiológica (SIVIGILA), dispone los datos de notificación obligatoria de casos. Esta entidad en el 2013 publicó, la prevalencia nacional de infectados por el virus, reportando una cifra de 95187 casos. ligualmente, para la semana epidemiológica número 22 de 2016 se han reportado 4998 nuevos casos de $\mathrm{VIH} / \mathrm{SIDA}^{9}$. Dentro de las estadísticas del Ministerio se calculó que para el 2009 había un prevalencia en la población general de $0,22 \%$ y cifras superiores al $5 \%$ en hombres que se relacionan sexualmente con hombres, mujeres trabajadoras sexuales, usuarios de drogas inyectables, adolescentes, mujeres gestantes, población privada de la libertad o en situación de desplazamiento forzoso y habitantes de la calle. Ciudades como Barranquilla, Cartagena y Medellín presentan cifras más altas que el promedio nacional en población de riesgo, bordeando el $13,6 \%, 10 \%$ y 9,1\% respectivamente $\mathrm{e}^{10-12}$.

El objetivo de este artículo es hacer una revisión de las principales complicaciones cardiovasculares y metabólicas desde un enfoque epidemiológico y fisiopatológico en pacientes infectados con $\mathrm{VIH}$ asociadas tanto a factores inherentes al virus como a su tratamiento con el fin de brindar una herramienta con respaldo científico al clínico, permitiendole un abordaje integral, periódico y oportuno con el fin de evitar otras casuas de muerte en los pacientes seropositivos.

\section{Metodología de búsqueda}

La información consignada en la presente revisión fue extraída luego de la búsqueda sistemática en las bases de datos PUBMED, EMBASE, CLNIICALKEY, 
OVID, REDALYC y MEDLINE durante los meses comprendidos entre enero y abril de 2016, utilizando como palabras clave términos MeSH de 2016: "Highly Active Antiretroviral Therapy", "Cardiovascular disease", "HIV infections" y "Metabolic Diseases". Se incluyeron metaanálisis, ensayos clínicos, artículos de revisión y artículos originales, publicados en los últimos diez años, que estuvieran en español o inglés. También se revisaron los informes realizados por el Programa Conjunto de las Naciones Unidas sobre el $\mathrm{VIH} / \mathrm{SIDA}$ (ONUSIDA) sobre estadísticas globales de la infección en 2000, 2013 y 2015, así mismo el Boletín Epidemiológico de la Semana 22 de 2016 publicado por la Dirección de Vigilancia y Análisis del Riesgo en Salud Pública del Ministerio de Salud de Colombia, también se revisó el documento Situación VIH/ SIDA en Colombia para el año 2013 publicado por el Ministerio de Salud de Colombia.

En total se otuvieron 119 articulos, los cuales fueron leídos llegando a descartar 49 de estos para obtener al final 70 artículos. Se tuvieron como criterios de exclusión la no disponibilidad del texto completo, idioma diferente a ingles y español, y revisiones de caso que enfatizaban en patologías no cardiometabólicas.

\section{Fisiopatología}

En pacientes con VIH se han demostrado múltiples mecanismos fisiopatológicos implicados en la alteración metabólica y cardiovascular, originados tanto por la infección por el VIH como por la TAR. Sin embargo, en los últimos años la investigación se ha dirigido a la comprensión del papel del sistema inmune y la inflamación en una compleja interacción de factores aún en estudio. Dentro de los mecanismos fisiopatológicos se pueden describir: toxicidad viral directa, infecciones oportunistas, respuesta del sistema inmune a la infección viral, cardiotoxicidad por medicamentos, deficiencias nutricionales e inmunosupresión prolongada ${ }^{4,13}$ (Ver Figura 1).

Existe una relación entre la respuesta inmune y la inflamación con un mayor riesgo de eventos cardiovasculares en pacientes infectados por $\mathrm{VIH}$. La inflamación se intensifica en aquellos pacientes sin un adecuado control de la infección, pero la evidencia apunta a que la inflamación crónica persiste aun en aquellos con adecuado tratamiento y supresión viral, lo que podría explicar las elevadas tasas de enfermedad cardiovascular a pesar de un adecuado tratamiento ${ }^{2}$. Este aumento de la respuesta inflamatoria igualmente se ha asociado con replicación viral activa, aterosclerosis acelerada, eventos cardiovasculares, aumento en la mortalidad y marcadores inflamatorios ${ }^{13}$.

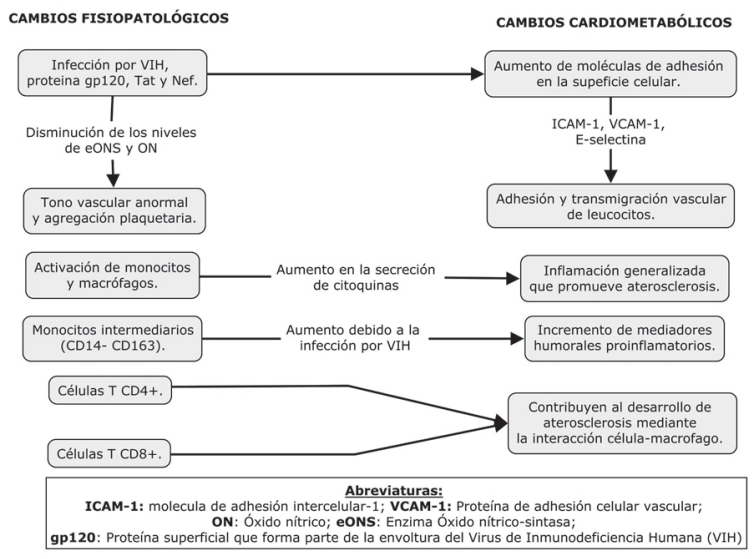

Figura 1. Procesos fisiopatológicos implicados en infección por VIH/SIDA.

Fuente: Zou W, Berglund L. HIV and highly active antiretroviral therapy: dyslipidemia, metabolic aberrations, and cardiovascular risk. Prev Cardiol. 2007;10(2):96-105.

Los marcadores inflamatorios se han estudiado como posibles herramientas predictivas de la enfermedad cardiovascular en pacientes con VIH. En una análisis del estudio SMART se analizó el valor predictivo de la proteína C-reactiva, Interleuquina (IL) 6 y dímero-D en relación con la morbilmortalidad cardiovascular, concluyendo que existía una asociación directa entre estos marcadores y un mayor riesgo de eventos cardiovasculares, independientemente de otros factores de riesgo ${ }^{13,15}$. Estudios posteriores mostraron que la interrupción de la TAR coincidía con un aumento significativo en los niveles séricos de IL-6 y dímero $D^{16}$. Igualmente se sabe que la células de la corteza cerebral y cardíacas se comportan como reservorio viral por extensos períodos, aún luego de la TAR, generando la liberación crónica de diversas citoquinas tales como el Factor de Necrosis Tumoral a, IL 1, 6, 10 y Endotelina 1 (ET-1), pudiendo ocasionar miocardiopatía y encefalopatía,13. Así mismo el daño generado al sistema nervioso autónomo por lesión neuronal, disminuye la funcionalidad miocárdica por aumento de la actividad adrenérgica, consumo de oxígeno y disminución de receptores beta adrenérgicos reduciendo la contractilidad miocárdica, aumentando la mortalidad por insuficiencia cardíaca congestiva'.

Otros mediadores de la inflamación han tenido relevancia en los últimos años, tal es el caso del CD14 soluble, un biomarcador de la activación de monocitos y macrófagos que se ha relacionado con 
aumento en la mortalidad cardiovascular y que en pacientes con VIH está aumentado ${ }^{17}$. Igualmente, el CD163 soluble, expresado por macrófagos en las placas ateroescleróticas, se correlaciona con el grado de inflamación arterial y se ha considerado como un potencial marcador de inflamación endotelial en pacientes con $\mathrm{VIH}^{16}$.

La lesión sobre las células endoteliales es otro mecanismo inflamatorio que se ha descrito, el cual se genera por la expresión de quimiocinas y proteínas de adhesión celular tales como ICAM-1, VCAM-1 y E-selectina que promueven el reclutamiento de monocitos y entrada de células inflamatorias en el endotelio lo cual aumenta el riesgo de eventos cardiovasculares. La evidencia de los ensayos clínicos, indica que este marcador está aumentado en pacientes con mal control de la enfermedad 3 . Sin embargo, el valor clínico de este marcador es aún incierto, en un estudio realizado en población eslovena no se encontró una concentración significativa de los niveles séricos de estas moléculas en pacientes con ateroesclerosis subclínica e infectados con $\mathrm{VIH}^{18}$.

En general, hay un creciente interés en la relación de los biomarcadores inflamatorios y las enfermedades cardiovasculares en la infección por $\mathrm{VIH}^{19}$. El uso de estos marcadores como medidas de comorbilidad para una mejor predicción de riesgo o intervenciones terapéuticas específicas aún no está claro, y se requiere determinar de manera más objetiva su verdadero uso clínico3.

Teniendo en cuanta la aplicación de TARGA y el riesgo cardiovascular previo al tratamiento contra el VIH, varios estudios alrededor del mundo dan evidencia de la estrecha relación de ambos y su impacto en la sobrevida de estos pacientes. En un estudio realizado por Mashinya y Colaboradores donde evaluaron los efectos adversos de la TAR, perfil lipídico, tabaquismo, obesidad, diabetes, carga viral y conteo de CD4, concluyeron que los factores de riesgo cardiovasculares fueron frecuentes en la población infectada con VIH en tratamiento y además una alta proporción de estos se encontraban en moderadoalto riesgo según la escala Framinghan ${ }^{13,20}$. Otro estudio realizado por Dickson y Colaboradores cuyo objetivo era investigar el perfil de riesgo cardiovascular de los pacientes VIH que recibían TARGA y de los que no la recibían, el resultado obtenido asoció el tratamiento de la TARGA con mayor prevalencia de hipercolesterolemia, aumento de colesterol LDL e hipertensión arterial ${ }^{21}$.

\section{COMPROMISO CARDIOVASCULAR EN PACIENTES CON VIH}

En los pacientes infectados con VIH, se han demostrado un gran número de manifestaciones y enfermedades cardiácas. Las de mayor importancia clínica y que han demostrado tener un mayor impacto en la morbimortalidad son tales como, la falla cardiaca, la Enfermedad Arterial Coronaria (EAC), el Infarto Agudo de Miocardio (IAM), el Ataque Cerebrovascular(ACV), la hipertensión arterial, la hipertensión pulmonar y las alteraciones metabólicas ${ }^{22}$.

\section{FALLA CARDÍACA}

La insuficiencia cardiaca ha sido una manifestación muy común entre las personas infectadas con VIH, donde su prevalencia no se ha visto afectado por disponibilidad de la TAR. Sin embargo, la compresión de la patogénesis de este proceso no se ha abordado de manera sistemática en estudios ${ }^{23}$. Hay diversas hipótesis que pueden ser sugestivas de este proceso de afección, entre estas están: replicación viral, infecciones oportunistas, influencia antirretroviral, entre otras causas. La falla cardiaca relacionada con pacientes VIH se produjo con mayor frecuencia en personas con un conteo de CD4 menor a 100 células por $\mathrm{mm}^{3}$, así mismo se presentó en pacientes con un nivel socioeconómico menor, pacientes en etapas avanzadas de la enfermedad y pacientes con cargas virales altas. Las tasas de mortalidad hospitalaria, en dos países africanos, por falla cardíaca asociada a VIH alcanzaron el 15\% para el año $2007^{24,25}$. En dos grandes estudios observacionales realizados en países de ingresos bajos y medios se informó que la falla cardíaca asociada a VIH aún es un diagnóstico muy común en estos pacientes siendo necesario una intervención más temprana²,23,26,27.

Una hipótesis implica la toxicidad miocárdica directa viral, donde el ácido ribonucleico del VIH en altas concentraciones, es decir más de 500 copias por $\mathrm{mL}$ de sangre, se asocia con cerca de 2,5 veces más probabilidad de desarrollar falla cardiaca en comparación con individuos no infectados ${ }^{27}$. La expresión transgénica de miocardio a través del activador de la transcripción del VIH (proteína Tat), activa las células endoteliales, provocando así la disfunción sistólica ventricular izquierda, generando hipertrofia ventricular izquierda y la expresión continua de péptidos natriuréticos perpetuados 
que dan lugar a un compromiso hemodinámico difuso ${ }^{28}$. Hoy en día aún es debatido la forma en que el virus entra al miocardiocito, la cual es una célula que es considerada reservorio viral pese a que no posee receptores $C D 4$. Estudios in vitro de miocitos cardiacos humanos y de rata muestran que el virus puede entrar a través de vías independientes de los receptores CCR5 o CXCR4 4,29,30.

Estudios recientes han encontrado la asociación entre el uso de antirretrovirales y toxicidad miocárdica ${ }^{31}$. Aunque la introducción de la terapia antirretroviral en países desarrollados se ha asociado con una reducción en la incidencia de falla cardíaca en pacientes $\mathrm{VIH}$ en un 30-50\%, la TARGA se ha implicado como causal de la disfunción ventricular sistólica izquierda32. La Zidovudina, un inhibidor de la transcriptasa inversa, se ha relacionado a miopatía esquelética y cardiaca reversible, atribuido a daño en la ultra estructura y ADN mitocondrial generando acidosis láctica que conlleva a disfunción de la célula miocárdica ${ }^{4}$. Estudios recientes encuentran evidencia suficiente para la relación entre la utilización de Zidovudina y disfunción diastólica33. Estos hallazgos son de suma importancia para los países de bajos y medianos ingresos donde la Zidovudina es todavía una opción de primera línea para el tratamiento del VIH para adultos, adolescentes y niños.

La autoinmunidad cardiaca es otra hipótesis estudiada desde los años 90. Currie y Cols., encontraron que autoanticuerpos cardiacos circulantes se detectan con más frecuencia en las persona con VIH y falla cardiaca que en los pacientes infectados con VIH sin alteración cardiaca o en los pacientes controles no infectados por $\mathrm{VIH}^{34}$.

\section{ENFERMEDAD ARTERIAL CORONARIA E INFARTO DE MIOCARDIO}

Los primeros casos de síndromes coronarios agudos en pacientes infectados con VIH fueron reportados en 199835. Grandes estudios observacionales de cohorte demostraron consistentemente mayores tasas de $E C V$, incluyendo IAM entre los infectados con VIH en comparación con los controles no infectados, con un aproximado de 1,5 a 2 veces mayor riesgo relativo en la última década ${ }^{36-41}$. Datos recientes han reforzado estos hallazgos, por ejemplo en un estudio de más de 27000 pacientes infectados con VIH, las tasas de IAM fueron mayores para pacientes VIH en todos los grupos etarios en comparación con los pacientes control emparejados ${ }^{39}$.
Una característica única de EAC asociada al VIH es la presencia de una forma histológicamente distintiva de aterosclerosis acelerada con una afectación circunferencial y difusa a lo largo de toda la arteria ${ }^{42,43}$, así como una proliferación inusual de las células de musculo liso, mezclada con abundantes fibras elásticas, lo que resulta en protrusiones endoluminales. Las células endoteliales parecen jugar un papel importante mediante la alteración de vías procoagulantes, anticoagulantes y fibrinoliticas ${ }^{44}$. La adhesión alterada de monocitos y macrófagos infectados con VIH y la vasculitis asociada al virus pueden ser otros factores que contribuyen a la arteriopatía coronaria ${ }^{43,44}$.

Varios estudios retrospectivos han vinculado la EAC con pacientes VIH que reciben TARGA, por ejemplo, Klein y Cols. reportaron una incidencia de eventos coronarios de 5,5 por 1000 pacientes-año en pacientes que recibían tratamiento, lo cual fue mayor que en el grupo control ${ }^{45}$. Se ha sugerido que el uso de Inhibidores de Proteasa (IP) y el uso reciente o actual de Abacavir puede aumentar hasta dos veces el riesgo de infarto por un aumento de la dislipidemia, la resistencia a la insulina, la activación plaquetaria, niveles aumentados de péptido $C$, la lipodistrofia y la disfunción endotelial|44,46.

La presentación angiográfica, el manejo y los resultados después de un evento cardiovascular agudo pueden diferir en los pacientes con $\mathrm{VIH}^{13}$. Se han documentado diferencias clínicas y angiográficas en la presentación de síndromes coronarios agudos en pacientes con $\mathrm{VIH}$, incluidas mayores tasas de reestenosis 47 y presentación más frecuente de IAM con elevación del segmento ST versus IAM sin elevación del segmento ST, o angina inestable en pacientes VIH frente a los controles ${ }^{48}$.

\section{ATAQUE CEREBRO VASCULAR}

La infección por el VIH predispone a los pacientes a una gran variedad de trastornos neurológicos, incluyendo el ACV. En series clínicas globales, entre $1 \%$ y el $5 \%$ de los pacientes con VIH desarrollaron un ACV, aunque una mayor proporción (4-34\%) presentaron lesiones isquémicas cerebrales halladas en la autopsia ${ }^{49}$.

La fisiopatología es probablemente multifactorial, entre los mecanismos propuestos están, la vasculitis infecciosa, la disfunción endotelial inducida por el VIH y los efectos adversos de la terapia antirretroviral 
combinada. Los individuos con ictus, que son VIH positivos, son pacientes más jóvenes que los individuos con ACV que no tienen la infección, lo que podría ser el resultado de la edad de la población en riesgo de infección por VIH o una señal de que el mecanismo de ACV en el VIH es en gran parte independiente de los factores de riesgo vasculares clásicos ${ }^{50}$. Sin embargo, la aparición de ictus y de la infección por VIH puede a menudo ser una coincidencia. La vasculopatía asociada al VIH describe diversos cambios cerebrovasculares, incluyendo estenosis y formación de aneurismas, vasculitis y la aterosclerosis acelerada, y podría ser causado directamente o indirectamente por la infección por VIH, aunque los mecanismos aún son controvertidos ${ }^{49}$.

La infección por VIH puede, potencialmente, causar un ACV por los siguientes mecanismos: indirectamente a través del cardioembolismo, coagulopatias o vasculitis infecciosa no relacionada con VIH, directamente el VIH podría provocar una vasculopatía (Ver Tabla 1).

Un estudio reciente sugiere que mientras que los factores de riesgo tradicionales son importantes para la patogénesis del ACV (en especial la hipertensión), la infección por el VIH, especialmente cuando no está bien controlada (es decir, recuentos menores de CD4 y mayor concentración de ARN VIH-1), contribuye al riesgo de ACV independientemente. Estos resultados tienen implicaciones importantes en el manejo de los pacientes que van envejeciendo con el VIH, y pueden ayudar a las estrategias terapéuticas para disminuir el riesgo de ictus isquémico en esta población de alto riesgo ${ }^{51}$.

\section{HIPERTENSIÓN ARTERIAL}

Los pacientes con VIH presentan mayor riesgo de desarrollar hipertensión arterial a edades más tempranas que la población general, siendo su prevalencia del 20 al 25\% antes de la introducción de la TARGA, esta situación es atribuida a factores predisponentes como la disfunción endotelial inducida por el virus, vasculitis de pequeños, medianos y grandes vasos, aneurismas de los grandes vasos con deterioro del flujo renal, insulinoresistencia, aumento de la actividad simpática y retención de sodio 4 . Así mismo, la hipertensión arterial se ha asociado frecuentemente con varias complicaciones relacionadas al VIH como resistencia adquirida a glucocorticoides, insuficiencia renal, vasculopatía, aterosclerosis e interacciones medicamentosas ${ }^{52}$.
Tabla 1. Posibles causas relacionadas con el vih de un accidente cerebrovascular $^{49}$.

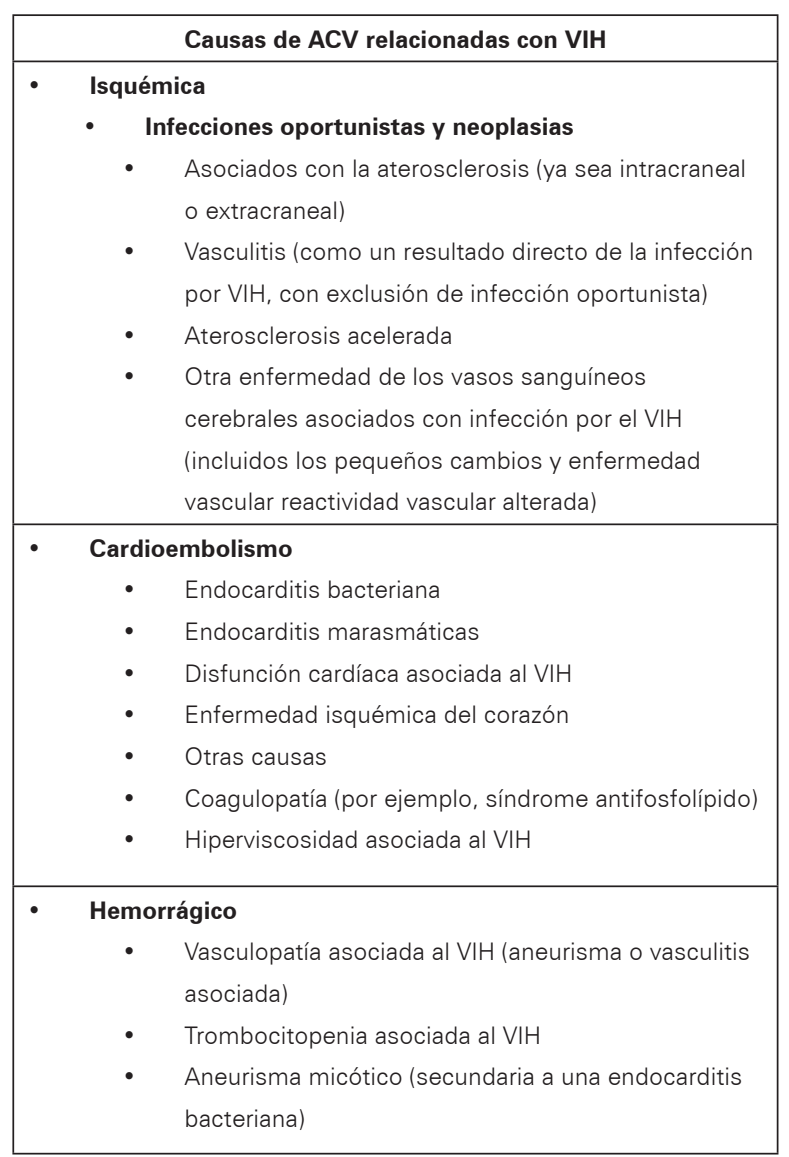

Fuente: Benjamin LA, Bryer A, Emsley HCA, Khoo S, Solomon T, Connor MD. HIV infection and stroke: Current perspectives and future directions. Lancet Neurol [Internet]. Elsevier Ltd; 2012;11(10):878-90. Available from: URL: http://dx.doi. org/10.1016/S1474-4422(12)70205-3

Una relación entre presión arterial sistólica elevada y duración del VIH se reportó en países con altos ingresos. Seaberg y Colaboradores ${ }^{53}$ en una cohorte multicéntrica de hombres seguidos entre 1984 y 2003 , reportaron que la mayor probabilidad de desarrollar hipertensión sistólica se daba después de cinco años o más de la instauración de la TARGA, especialmente en regímenes basados en IP inhibidores no nucleósidos de la transcriptasa inversa. Igualmente, se demostró que la incapacidad de la presión arterial de disminuir mas del 10\% durante la noche, un factor de riesgo de eventos cardiovasculares, es más común en los pacientes con VIH ${ }^{54}$.

Por otra parte, en los países en vías de desarrollo la prevalencia y severidad de la hipertensión en pacientes VIH positivos ha ganado atención recientemente. Estudios realizados en Kenia y Uganda reportan tasas de prevalencia de hipertensión entre 11 y $28 \%$ 
entre los pacientes VIH positivos y los hombres se ven afectados de manera desproporcionada. Sin embargo, son necesarios datos prospectivos para esclarecer esta relación ${ }^{55,56}$.

\section{HIPERTENSIÓN PULMONAR}

Desde que se describió el primer caso de Hipertensión Pulmonar Arterial (HPA) en VIH/SIDA en 1987, se ha reconocido esta entidad como una de las complicaciones más severas de la infección por VIH llevando a una alta mortalidad, donde la hipertensión pulmonar fue la causa directa de muerte en el $72 \%$ de los $\operatorname{casos}^{22,57}$. La más reciente clasificación de Niza estadifica la HPA generada por el VIH como tipo I, es decir la que compromete exclusivamente el lecho $\operatorname{arterial}^{58}$.

La prevalencia en los países desarrollados es baja (<1\%), sin embargo, en algunos países en vía de desarrollo ocupa el segundo lugar como causa de HPA. La edad promedio de presentación es de 33 años con mayor incidencia en sexo femenino ${ }^{59,60}$. La prevalencia de la HPA no ha tenido grandes cambios pese a la introducción de la TAR, además que no existe una correlación entre el grado de inmunosupresión y la gravedad de presentación ${ }^{61}$. La supervivencia en los pacientes con HPA asociada a VIH es menos favorable en comparación con pacientes VIH sin esta asociación o con HPA idiopática ${ }^{17,18}$.

No existe evidencia de una infección directa a las células endoteliales pulmonares que pudiera explicar el compromiso vascular, sin embargo, antígenos virales están presentes en el endotelio vascular que pueden estimular directamente una apoptosis anormal, el crecimiento y la proliferación celular. Además, la infección por VIH induce un estado inflamatorio crónico que se caracteriza por la activación inmunitaria persistente que podría inducir indirectamente la liberación de citoquinas proinflamatorias y factores de crecimiento que podrían producir HPA ${ }^{62}$. Las proteínas Nef y Tat del VIH contribuyen a procesos claves de vasoconstricción, proliferación celular y trombosis. En la envoltura del VIH la glicoproteína Gp 120, estimula los macrófagos y las células endoteliales arteriales en el pulmón a secretar ET-1, un potente péptido vasoconstrictor con efectos proliferativos y pro-inflamatorios ${ }^{63}$. En un estudio realizado por Feijoo y cols. se demostró que los pacientes VIH positivo presentaban mayor nivel sérico de ET-1 en comparación con el control, esto se correlacionó con una mayor presión arterial pulmonar sistólica y podría considerarse un marcador precoz de disfunción del ventrículo derecho ${ }^{64}$.

\section{ALTERACIONES METABÓLICAS}

Lasalteracionesmetabólicas tienenunaaltaincidencia entre los pacientes con VIH/SIDA en todo el mundo. La dislipidemia, la lipodistrofia y la resistencia a la insulina son las principales enfermedades que están presentes en estos pacientes ${ }^{65}$. Dados los procesos fisiopatológicos mencionados, estas enfermedades tienen una íntima relación en su historia natural (Ver Figura 2) por lo cual en la mayoría de los pacientes infectados se presentan de manera simultánea y progresiva $^{14}$.

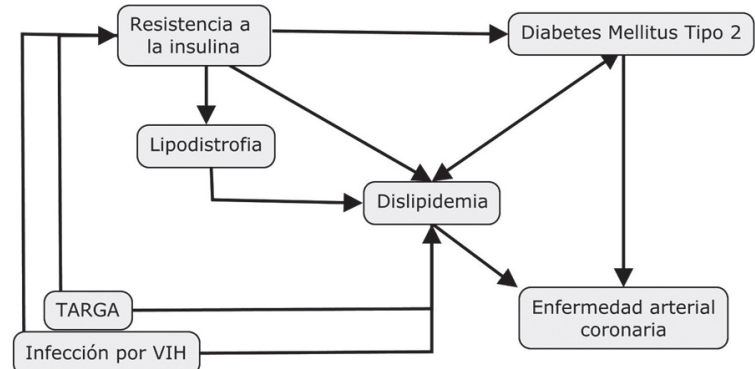

Figura 2. ALTERACIONES METABOLICAS EN PACIENTES VIH/SIDA. Fuente: Zou W, Berglund L. HIV and highly active antiretroviral therapy: dyslipidemia, metabolic aberrations, and cardiovascular risk. Prev Cardiol. 2007; 10(2):96-105.

La dislipidemia asociada al VIH es un problema que se estima se presenta en un $48 \%$ y se asocia con aumento en la incidencia de la enfermedad cardiovascular ${ }^{66}$. Los mecanismos que han intentado explicar este fenómeno incluyen incremento en los niveles de citoquinas (TNF e IL-6), disminución de la depuración de los lípidos, y el aumento de la síntesis hepática de lipoproteína de muy baja densidad ${ }^{65}$. Diversos estudios han demostrado que existe un riesgo significativamente mayor de presentar dislipidemia en pacientes con VIH expuestos a tratamiento antirretroviral en comparación a quienes no lo recibieron, manifestada por lo general como disminución de HDL-c, aumento de LDL-c y aumento de colesterol total, un perfil lipídico similar al que se presenta en pacientes con síndrome metabólico 14,65,67. Dentro de los diferentes regímenes antirretrovirales, la dislipidemia se asocia en un 70\% los que incluyen los inhibidores de la proteasa, igualmente se relacionan con obesidad central, lipodistrofia y resistencia a la insulina $^{65}$. Por lo que algunos autores recomiendan en todo adulto mayor infectado por VIH la medición de perfil lipídico cada año antes del inicio de TAR y 
uno o dos meses después de cualquier cambio en el régimen antirretroviral ${ }^{68}$.

La intolerancia a la glucosa se estima que está presente en cerca del 35\% de los sujetos infectados por el VIH en comparación con el $5 \%$ de los sujetos de control sanos ${ }^{14,69}$. Se estima que riesgo de desarrollar diabetes mellitus tipo 2 es 3,1 veces mayor en pacientes con VIH que reciben TAR en comparación con sujetos sin tratamiento ${ }^{70}$. La TAR da lugar a un flujo alterado de sustratos incluyendo ácidos grasos libres, así como acumulación de lípidos intramiocelulares y alteración de los niveles de adipocinas; además puede contribuir a una homeostasis alterada de la glucosa. Los IP (incluyendo Ritonavir, Nelfinavir), han demostrado inducir resistencia a la insulina in vivo mediante la reducción del transporte de glucosa mediada por el transportador de glucosa 4 (GLUT-4) y disminución de la secreción de insulina por parte de las células beta pancreáticas. Ademas, se observó restauración de la sensibilidad a la insulina después de la restricción al medicamento ${ }^{67,69}$. Por lo anterior, recomiendan la realización de una prueba de tolerancia oral a la glucosa o medición de los niveles de insulina en ayunas en pacientes infectados con $\mathrm{VIH}$ con otros factores de riesgo cardiovascular o antecedentes familiares de diabetes mellitus tipo $2^{68}$.

El síndrome de lipodistrofia asociada a VIH incluye lipoatrofia y lipohipertrofia. La lipoatrofia usual en cara, extremidades inferiores y glúteos que está estrechamente relacionada con el uso de análogos de nucleósidos que actúan como inhibidores de la transcriptasa inversa y rara vez se asocia con IP. Por otro lado, la lipohipertrofia está relacionada con la acumulación de tejido adiposo preferiblemente en abdomen, pecho y región interescapular y probablemente asociada al uso de IP. Dentro de los mecanismos que explican el desarrollo de esta entidad se encuentran el proceso de inflamatorio generalizado, alteración del metabolismo de lípidos por inhibición de la lipoproteina lipasa y el exceso de triglicéridos, el estrés oxidativo y la alteración de la función de las adipoquininas ${ }^{16}$.

\section{CONCLUSIONES}

La ECV asociada al VIH es causada por una compleja interacción de factores en relación con el virus, el huésped y la terapia antirretroviral. El adecuado conocimiento de los mecanismos involucrados y su repercusión en la salud del paciente se deben tener presentes para estimar los riesgos y beneficios de las intervenciones medicas.

En la revisión realizada de la literatura, se encontraron diversas manifestaciones cardiovasculares que empeoran la calidad de vida de los pacientes VIH seropositivos, estas son: la falla cardiaca, EAC, IAM, $\mathrm{ACV}$, hipertensión arterial, hipertensión pulmonar y alteraciones metabólicas (Dislipidemias), las cuales son las nuevas causas de muerte en estos pacientes, por encima de la muerte directamente relacionada por el estadio final de SIDA.

Se espera que en los próximos años el riesgo de ECV asociada al VIH aumente ${ }^{22}$, es por esto que se deberán implementar medidas dirigidas a la prevención y modificación de los factores de riesgo, además de plantear y evaluar nuevas estrategias de tamizaje e intervenciónes terapéuticas dirigida a reducir y prevenir los procesos fisiopatológicos que son los principales implicados en el desarrollo de la enfermedad cardiometabólica, haciendo un abordaje integral al paciente. Queda un amplio campo por explorar aún, partiendo de la existencia de nuevas formas de presentación de una enfermedad ya conocida.

\section{RefERENCIAS BibLIOGRÁfICAS}

1. Henry K, Melroe H, Huebsch J, Hermundson J, Levine C, Swensen $\mathrm{L}$, et al. Severe premature coronary artery disease with protease inhibitors. Lancet. 1998;351(9112):1328.

2. Hemkens LG, Bucher HC. HIV infection and cardiovascular disease. Eur Heart J. 2014;35(21):1373-81.

3. Hickok J, Avery TR, Lankiewicz J, Gombosev A, Terpstra L, Hartford F, et al. CD4+ Count-Guided Interruption of Antiretroviral Treatment. NEJM. 2006;355(22):2255-65.

4. Risso GD. Enfermedad cardiovascular en sujetos con VIH / SIDA Cardiovascular disease in patients with HIV / AIDS. Rev Fed Arg Cardiol. 2012;41(4):235-48.

5. ONUSIDA. Informe Mundial: ONUSIDA, Informe sobre la epidemia mundial de SIDA. 2013. p. 274.

6. Programa Conjunto de las Naciones Unidas sobre el VIH/SIDA. Hoja informativa 2015. 2015; p. 1-8.

7. Programa Conjunto de las Naciones Unidas sobre el VIH/SIDA. El SIDA en cifras 2015. 2015; p. 1-12.

8. Teva I, Bermúdez MP, Ramiro MT, Buela-Casal G. Situación epidemiológica actual del VIH/SIDA en Latinoamérica en la primera década del siglo XXI: Análisis de las diferencias entre países. Rev Med Chil. 2012;140(1):50-8.

9. Ministerio de Salud y Protección Social de Colombia. Boletin Epidemiológico semanal. Semana epidemiológica número 22 de 2016. Colombia; 2016;2016.

10. Ministerio de Salud y Protección Social de Colombia. Resumen de situación de la epidemia por VIH / SIDA en Colombia 1983 a 2011. 2012; p. 1-15.

11. Instituto Nacional de Salud I, Ministerio de Salud y Proteccion Social. Protocolo de vigilancia en Salud Pública. VIH - SIDA. 2014. p.43.

12. Nuñez RL, Paola S, Mira Á. Boletín epidemiológico, situación del VIH / SIDA Colombia 2013. 2013.

13. Triant VA. Cardiovascular disease and HIV infection. Curr HIV/ 
AIDS Rep. 2013;10(3):199-206.

14. Zou W, Berglund L. HIV and highly active antiretroviral therapy: dyslipidemia, metabolic aberrations, and cardiovascular risk. Prev Cardiol. 2007;10(2):96-105.

15. Duprez DA, Neuhaus J, Kuller LH, Tracy R, Belloso W, De Wit S, et al. I nflammation, coagulation and cardiovascular disease in HIV-infected individuals. PLOS ONE. 2012;7(9):e44454.

16. Hemkens LG, Bucher HC. HIV infection and cardiovascular disease. Eur Heart J. 2014;35(21):1373-81.

17. Sandler NG, Wand H, Roque A, Law M, Nason MC, Nixon DE, et al. Plasma Levels of Soluble CD14 Independently Predict Mortality in HIV Infection. J Infect Dis. 2011;203(6):780-90.

18. Pirš M, Jug B, Eržen B, Šabović M, Karner P, Poljak M, et al. Relationship between markers of endothelial dysfunction and inflammation and subclinical atherosclerosis in HIV-infected male patients below 55 years of age. Acta Dermatovenerologica Alp Pannonica Adriat. 2014;23(3):49-52

19. Hsue PY, Ordovas K, Lee T, Reddy G, Gotway M, Schnell A, et al. Carotid Intima-Media Thickness Among Human Immunodeficiency Virus-Infected Patients Without Coronary Calcium. Am J Cardiol. 2012;109(5):742-7.

20. Mashinya F, Alberts M, Van geertruyden J-P, Colebunders R. Assessment of cardiovascular risk factors in people with HIV infection treated with ART in rural South Africa: a cross sectional study. AIDS Res Ther. BioMed Central. 2015;12(1):42.

21. Nsagha DS, Assob JCN, Njunda AL, Tanue EA, Kibu OD, Ayima CW, et al. Risk Factors of Cardiovascular Diseases in HIV/AIDS Patients on HAART. Open AIDS J. 2015;9(1):51-9.

22. Bloomfield GS, Khazanie P, Morris A, Rabadán-Diehl C, Benjamin LA, Murdoch D, et al. HIV and Noncommunicable Cardiovascular and Pulmonary Diseases in Low- and Middle-Income Countries in the ART Era: What We Know and Best Directions for Future Research. J Acquir Immune Defic Syndr. 2014;67 Suppl 1:S4053.

23. Bloomfield GS, Alenezi F, Barasa FA, Lumsden R, Mayosi BM, Velazquez EJ. Human Immunodeficiency Virus and Heart Failure in Low- and Middle-Income Countries. JACC Heart Fail 2015;3(8):579-90.

24. Twagirumukiza M, Nkeramihigo E, Seminega B, Gasakure E, Boccara F, Barbaro G. Prevalence of dilated cardiomyopathy in HIV-infected African patients not receiving HAART: a multicenter, observational, prospective, cohort study in Rwanda. Curr HIV Res. 2007;5(1):129-37.

25. Nzuobontane D, Blackett KN, Kuaban C. Cardiac involvement in HIV infected people in Yaounde, Cameroon. Postgrad Med J. 2002;78(925):678-81

26. Shah MR. The Broad Spectrum of HIV-Related Cardiovascular Disease*. JACC Heart Fail. Elsevier Inc; 2015;3(8):600-2.

27. Butt AA, Chang C-C, Kuller L, Goetz MB, Leaf D, Rimland D, et al. Risk of Heart Failure With Human Immunodeficiency Virus in the Absence of Prior Diagnosis of Coronary Heart Disease. Arch Intern Med. 2011;171(8):737-43.

28. Duan M, Yao H, Hu G, Chen XM, Lund AK, Buch S. HIV Tat Induces Expression of ICAM-1 in HUVECs: Implications for miR-221/-222 in HIV-Associated Cardiomyopathy. PLoS One. 2013;8(3):1-11

29. Twu C, Liu NQ, Popik W, Bukrinsky M, Sayre J, Roberts J, et al. Cardiomyocytes undergo apoptosis in human immunodeficiency virus cardiomyopathy through mitochondrion- and death receptor-controlled pathways. Proc Natl Acad Sci U S A. 2002:99(22):14386-91

30. Fiala M, Popik W, Qiao JH, Lossinsky AS, Alce T, Tran K, et al. HIV-1 induces cardiomyopathyby cardiomyocyte invasion and gp120, Tat, and cytokine apoptotic signaling. Cardiovasc Toxicol. 2004;4(2):97-107.

31. Currie PF, Boon NA. Immunopathogenesis of HIV-related heart muscle disease: Current perspectives. AIDS. 2003;17 Suppl 1:S21-8.

32. Pugliese A, Isnardi D, Saini A, Scarabelli T, Raddino R, Torre D. Impact of Highly Active Antiretroviral Therapy in HIV-positive Patients with Cardiac Involvement. J Infect. 2000 May;40(3):282-4.

33. Luo L, Ye Y, Liu Z, Zuo L, Li Y, Han Y, et al. Assessment of cardiac diastolic dysfunction in HIV-infected people without cardiovascular symptoms in China. Int J STD AIDS. 2010;21(12):814-8.

34. Currie PF, Goldman JH, Caforio AL, Jacob AJ, Baig MK, Brettle
RP, et al. Cardiac autoimmunity in HIV related heart muscle disease. Heart. 1998;79(6):599-604.

35. Patel VB, Sacoor Z, Francis P, Bill PLA, Bhigjee AI, Connolly C. Ischemic stroke in young HIV-positive patients in Kwazulu-Natal, South Africa. Neurology. 2005;65(5):759-61.

36. Martin-Iguacel R, Llibre JM, Friis-Moller N. Risk of Cardiovascular Disease in an Aging HIV Population: Where Are We Now? Curr HIV/AIDS Rep. 2015;12(4);375-87.

37. Triant VA, Lee H, Hadigan C, Grinspoon SK. Increased acute myocardial infarction rates and cardiovascular risk factors among patients with human immunodeficiency virus disease. J Clin Endocrinol Metab. 2007,92(7):2506-12.

38. Durand M, Sheehy O, Baril J-G, Lelorier J, Tremblay CL. Association between HIV infection, antiretroviral therapy, and risk of acute myocardial infarction: a cohort and nested casecontrol study using Québec's public health insurance database. J Acquir Immune Defic Syndr. 2011;57(3):245-53.

39. Freiberg MS, Chang C-CH, Kuller LH, Skanderson M, Lowy E, Kraemer KL, et al. HIV infection and the risk of acute myocardial infarction. JAMA Intern Med. 2013;173(8):614-22.

40. Silverberg MJ, Leyden WA, Xu L, Horberg MA, Chao CR, Towner WJ, et al. Immunodeficiency and Risk of Myocardial Infarction Among HIV-Positive Individuals With Access to Care. JAIDS J Acquir Immune Defic Syndr. 2014;65(2):160-6.

41. Paisible A-L, Chang C-CH, So-Armah KA, Butt AA, Leaf DA, Budoff M, et al. HIV infection, cardiovascular disease risk factor profile, and risk for acute myocardial infarction. J Acquir Immune Defic Syndr. 2015;68(2):209-16.

42. Tabib A, Leurox C, Mornex J LR. Accelerated coronary atherosclerosis and arteriosclerosis in young humanimmunodeficiency- virus-positive patients. Coron Artery Dis. 2000;11(1):41-6.

43. Mehta NJ KI. HIV-associated coronary artery diseas. Angiology. 2003;54(3):269-75.

44. Khunnawat C, Mukerji S, Havlichek D, Touma R, Abela GS. Cardiovascular manifestations in human immunodeficiency virus-infected patients. Am J Cardiol. 2008;102(5):635-42.

45. Klein D, Hurley LB, Quesenberry CP Jr SS. Do protease inhibitors increase the risk for coronary heart disease in patients with HIV-1 infection?. J Acquir Immune Defic Syndr. 2002;30(5):471-7.

46. Bavinger C, Bendavid E, Niehaus K, Olshen RA, Olkin I, Sundaram $\mathrm{V}$, et al. Risk of cardiovascular disease from antiretroviral therapy for HIV: a systematic review. PLoS One. 2013;8(3):e59551

47. Hsue PY, Giri K, Erickson S, MacGregor JS, Younes N, Shergill A, et al. Clinical features of acute coronary syndromes in patients with human immunodeficiency virus infection. Circulation. 2004;109(3):316-9.

48. Perello R. Clinical presentation of acute coronary syndrome in HIV infected adults: a retrospective analysis of a prospectively collected cohort. Eur J Int Med. 2011;22(5):485-8.

49. Benjamin LA, Bryer A, Emsley HCA, Khoo S, Solomon T, Connor MD. HIV infection and stroke: Current perspectives and future directions. Lancet Neurol. 2012;11(10):878-90.

50. Edwards NJ, Grill MF, Choi HA, Ko NU. Frequency and Risk Factors for Cerebral Arterial Disease in a HIV/AIDS Neuroimaging Cohort. Cerebrovasc Dis [Internet]. 2016;41(3-4):170-6. Available from: URL: http://www.karger.com/?doi=10.1159/000442755

51. Sico JJ, Chang CC, So-Armah K, Justice AC, Hylek E, Skanderson $\mathrm{M}$, et al. HIV status and the risk of ischemic stroke among men. Neurology. 2015;84(19):1933-40.

52. Aoun S, Ramos E. Hypertension in the HIV-infected patient. Curr Hypertens Rep. 2000;2(5):478-81.

53. Seaberg EC, Muñoz A, Lu M, Detels R, Margolick JB, Riddler SA, et al. Association between highly active antiretroviral therapy and hypertension in a large cohort of men followed from 1984 to 2003. AIDS. 2005;19(9):953-60.

54. De Socio GVL, Bonfanti P, Martinelli C, Ricci E, Pucci G, Marinoni M, et al. Negative influence of HIV infection on daynight blood pressure variability. J Acquir Immune Defic Syndr. 2010;55(3):356-60.

55. Bloomfield GS, Hogan JW, Keter A, Sang E, Carter EJ, Velazquez EJ, et al. Hypertension and obesity as cardiovascular risk factors among HIV seropositive patients in Western Kenya. PLoS One. 2011;6(7):e22288

56. Mateen FJ, Kanters S, Kalyesubula R, Mukasa B, Kawuma E, 
Kengne AP, et al. Hypertension prevalence and Framingham risk score stratification in a large HIV-positive cohort in Uganda. J Hypertens. 2013;31(7):1372-8.

57. Maur R, Bortman G. Hipertensión arterial pulmonar asociada a infección por el virus de la inmunodeficiencia humana. Insuf Card. 2007;2(1):41-2.

58. Simonneau G, Gatzoulis MA, Adatia I, Celermajer D, Denton C, Ghofrani A, et al. Updated clinical classification of pulmonary hypertension. J Am Coll Cardiol. 2013;62(25 Suppl):D34-41.

59. Morris A, Crothers K, Beck JM, Huang L. An official ATS workshop report: Emerging issues and current controversies in HIV-associated pulmonary diseases. Proc Am Thorac Soc. 2011;8(1):17-26.

60. Reinsch N, Buhr C, Krings P, Kaelsch H, Kahlert P, Konorza T, et al. Effect of gender and highly active antiretroviral therapy on HIV-related pulmonary arterial hypertension: Results of the HIV HEART Study. HIV Med. 2008;9(7):550-6.

61. Bloomfield GS, Khazanie P, Morris A, Rabadán-Diehl C, Benjamin LA, Murdoch D, et al. HIV and Noncommunicable Cardiovascula and Pulmonary Diseases in Low- and Middle-Income Countrie in the ART Era. J Acquir Immune Defic Syndr. 2014;67(Suppl 1):S40-53.

62. Barnett CF Hsue PY Human Immunodeficiency VirusAssociated Pulmonary Arterial Hypertension. Clin Chest Med. 2013;34(2):283-92.

63. Parikh RV, Ma Y, Scherzer R, Heringer AS, Macgregor JS, Martin
JN, et al. Endothelin-1 Predicts Hemodynamically Assessed Pulmonary Arterial Hypertension in HIV Infection. PLoS One. 2016;11(1):e0146355.

64. Quezada M, Toro R, López M, Lennie V, Eulalia Valencia LP de I y LMC. La Endotelina -1 y la función del ventrículo derecho: Nuevo marcador en población VIH positivo con Hipertensión Arterial Pulmonar. Rev Española Cardiol. 2014;67(Supl 1:885):2-3.

65. Husain NE, Ahmed M. Managing dyslipidemia in HIV/AIDS patients: challenges and solutions. HIV/AIDS. 2014;7:1-10.

66. Chu C, Umanski G, Blank A, Meissner P, Grossberg R, Selwyn PA. Comorbidity-related treatment outcomes among HIV-Infected adults in the Bronx, NY. J Urban Health. 2011;88(3):507-16.

67. Riddler SA, Smit E, Cole SR, Li R, Chmiel JS, Dobs A, et al. Impact of HIV infection and HAART on serum lipids in men. JAMA. 2003;289(22):2978-82.

68. Grinspoon S, Carr A. Cardiovascular risk and bodyfat abnormalities in HIV-infected adults. N Engl J Med. 2005;352(1):48-62.

69. Hadigan C, Meigs JB, Corcoran C, Rietschel P, Piecuch S, Basgoz $\mathrm{N}$, et al. Metabolic Abnormalities and Cardiovascular Disease Risk Factors in Adults with Human Immunodeficiency Virus Infection and Lipodystrophy. Clin Infect Dis. 2001;32:130-9.

70. Petoumenos K, Worm SW, Fontas E, Weber R, De Wit S, Bruyand $\mathrm{M}$, et al. Predicting the short-term risk of diabetes in HIV-positive patients: The Data Collection on Adverse Events of Anti-HIV Drugs (D:A:D) study. J Int AIDS Soc. 2012;15(2):1-10. 